\title{
Glycoprotein non-metastatic melanoma protein B as a predictive prognostic factor in clear-cell renal cell carcinoma following radical nephrectomy
}

\author{
CAIPENG QIN $^{1 *}$, ZHENHUA LIU $^{1 *}$, YEQING YUAN $^{1}$, XIAOWEI ZHANG ${ }^{1}$, \\ HENAN LI ${ }^{2}$, CHUNFANG ZHANG ${ }^{3}$, TAO XU ${ }^{1}$ and XIAOFENG WANG ${ }^{1}$ \\ Departments of ${ }^{1}$ Urology, ${ }^{2}$ Clinical Laboratory and ${ }^{3}$ Scientific Research, \\ Peking University People's Hospital, Beijing 100044, P.R. China \\ Received September 5, 2013; Accepted January 9, 2014
}

DOI: $10.3892 / \mathrm{mmr} .2014 .1896$

\begin{abstract}
Current cancer therapies are largely reliant upon drugs and radiation that kill dividing cells or inhibit cell division in the primary tumor after radical surgery, whereas metastatic tumors should be more commonly the focus of targeted molecular therapies. Glycoprotein non-metastatic melanoma protein B (Gpnmb) is expressed in various types of cancer, and promotes the migration, invasion and metastasis of tumor cells. Thus, it was hypothesized that a unique clear-cell renal carcinoma (ccRCC) subclone expressing a high level of Gpnmb may metastasize more easily and thus be associated with poor prognosis. In the present study, the expression of Gpnmb was analyzed in primary and metastatic ccRCC samples, and the expression levels of Gpnmb were significantly higher in metastatic ccRCCs than in matched primary samples $(\mathrm{P}=0.036)$. Receiver operating characteristic (ROC) curve analysis was then performed to determine a cutoff score for Gpnmb expression in another 43 primary ccRCCs. For validation, the ROC-derived cutoff score was subjected to an analysis of the association between Gpnmb expression and patient outcome/clinical characteristics. Kaplan-Meier analysis demonstrated that elevated Gpnmb expression predicted a poorer overall survival (OS) in 43 cases of primary ccRCC. In addition, multivariate analyses revealed that Gpnmb expression served as an independent risk factor for ccRCC. Thus, the overexpression of Gpnmb identified patients at high risk and Gpnmb is a potential therapeutic molecular target for this type of tumor.
\end{abstract}

Correspondence to: Professor Tao Xu, Department of Urology, Peking University People's Hospital, 11 Xizhimen South Street, Beijing 100044, P.R. China

E-mail:xutao@medmail.com.cn

*Contributed equally

Key words: clear-cell renal cell carcinoma, metastasis, glycoprotein non-metastatic melanoma protein $\mathrm{B}$, prognosis

\section{Introduction}

Renal cell carcinoma (RCC) accounts for $2-3 \%$ of all adult cancers, is more common in males than females (2:1) and most commonly occurs in patients aged 50-70 years (1). The incidence of RCC has increased and RCC is the most lethal of all urological malignancies (2). The development of macroscopic metastases is the predominant cause of tumor-associated mortality $(3,4)$. For RCC metastatic disease affects one-third of patients at diagnosis and an additional one-third develop metastatic disease following radical nephrectomy (5). Patients who present with metastatic disease have a median survival time of 7-11 months and a 5-year mortality rate of $>90 \%$ (6). Additionally, clear-cell RCC (ccRCC) is the most common type (70-80\%) of RCC metastatic disease (7). Therefore, molecular markers are urgently required to predict individual metastatic risk and prognosis in ccRCC patients. Such markers represent the prerequisite for optimal follow-up and treatment of patients following surgery.

Glycoprotein non-metastatic melanoma protein B (Gpnmb) is a transmembrane glycoprotein that is expressed in various types of cancer. This protein was first cloned from a melanoma cell line and is expressed at a high level in a number of melanoma cell lines (8). The gpnmb gene is located on human chromosome $7 \mathrm{q} 15$ and encodes a type I transmembrane protein that is expressed in a wide variety of human tissues and cells, including the embryonic nervous system, developing nephrons, the germinal cells of hair follicles, osteoblasts, osteoclasts, myocytes, retinal pigment epithelium, renal tubules, macrophages and dendritic cells (9-11). The Gpnmb protein consists of at least four domains: An N-terminal domain with a signal peptide, a polycystic kidney disease domain, a transmembrane domain and an ARG-GLY-ASP domain. The Arg-Gly-Asp (RGD) cell attachment domain is for integrin-mediated cell attachment and migration. Further analysis has suggested that Gpnmb is highly glycosylated and has two isoforms: A secreted type and a transmembrane type. Moreover, the expression of Gpnmb is directly regulated by microphthalmia-associated transcription factor (12). Using immunohistochemistry, Hong et al (8) were the first to report Gpnmb expression in several cases of RCC. Since Gpnmb promotes the migration, 
invasion and metastasis of tumor cells, this protein represents a potential immunomarker for RCC.

Previous studies have revealed that the differential expression of proteomic markers in primary tumors and metastatic ccRCC tissues is important in drug resistance (1,5,13-15). Furthermore, poor prognoses and therapeutic outcomes may be attributed to a high level of these molecules, which are associated with metastasis. To the best of our knowledge, no studies have evaluated the difference in Gpnmb expression between primary and metastatic ccRCCs. In the present study, ccRCC specimens were selected according to a strict protocol to detect differences in Gpnmb expression levels between primary and matched metastatic ccRCC samples. In addition, the prognostic significance and therapeutic potential of these differential expression levels were analyzed.

\section{Materials and methods}

Patient and tumor characteristics. Two tissue microarrays were constructed. The first array consisted of primary ccRCCs and their matched metastases $(n=12)$, including seven ccRCCs with bone metastases, one with lung metastases, two with adrenal metastases and two with lymph node metastases near the inferior vena and/or renal hilus. The second array consisted of a subset that included only primary ccRCC samples $(n=43)$. Twelve ccRCC patients from the archives of the Department of Pathology at the People's Hospital of Peking University (Beijing, China) were initially recruited for the present study according to a protocol that was approved by the Institutional Review Board. The clinicopathological characteristics of the patients are summarized in Table I. The second array was designed by Shanghai Outdo Biotech Co., Ltd. (Shanghai, China) and contained material from 43 primary ccRCC patients. Clinical follow-up data were available for 43 patients who had undergone nephrectomy. The follow-up duration was calculated as the interval in months between initial presentation and either the final visit or RCC-related mortality. The length of the follow-up time ranged from 1-74 months. At the end of follow-up, 14 patients had succumbed to disease progression.

Immunohistochemical analysis and evaluation. Immunohistochemistry was performed using the Envision ${ }^{\mathrm{TM}}$ ABC kit (Dako, Capinteria, CA, USA) according to the manufacturer's instructions. After washing in phosphate-buffered saline, the tissue microarray and slides were incubated in $0.3 \%$ hydrogen peroxide for $10 \mathrm{~min}$ to quench endogenous peroxidase activity. The tissues were then pre-incubated with goat serum and incubated overnight at $4^{\circ} \mathrm{C}$ in a humidified chamber with a primary antibody against Gpnmb (monoclonal mouse; 1:100; MAB25501, R\&D Systems, Minneapolis, MN, USA). The primary antibody was detected using the Envision ${ }^{\mathrm{TM}}$ $\mathrm{ABC}$ kit. The color was developed with diaminobenzidine. Following counterstaining with hematoxylin, the sections were dehydrated and mounted. Gpnmb expression was scored as positive only upon observation of membranous staining and was measured using the $\mathrm{H}$-score [intensity (1, 2, 3 or 4) plus the distribution (\%)]. The expression was divided into three groups according to positive distribution of the tumor cell membranes: $1,0-5 \% ; 2,6-50 \%$; and $3,>50 \%$. To assess
Table I. Patient characteristics of the 12 paired renal cell carcinoma cases.

\begin{tabular}{lc}
\hline Characteristic & Variable \\
\hline Case number & 12 \\
Age (years), median (range) & $53.5(21-74)$ \\
Gender, male/female & $9 / 3$ \\
Pathological findings & \\
Clear cell type, $\mathrm{n}$ & 12 \\
Fuhrman grade, $\mathrm{n}$ & \\
$\leq \mathrm{II}$ & 7 \\
$>$ II & 5 \\
\hline
\end{tabular}

intratumor heterogeneity, three distinct microscopic fields (magnification, x200) per specimen were used to evaluate the expression of Gpnmb with a Leica DM400B (Leica Microsystems, Wetzlar, Germany), using a DM2500 image analysis system (exposure value, $16.5 \mathrm{~ms}$; saturation, 1.75; Gamma, 0.95; Gain, 1.0). Subsequently, the scores were averaged to obtain a single concatenated score for each tissue for Gpnmb expression. Gpnmb expression was assessed by two independent pathologists who were blinded to the clinicopathological data. The data are expressed as the mean value of the triplicate experiments.

Selection of cutoff score for positive Gpnmb expression. The selection of clinically important cutoff scores for Gpnmb expression was determined by receiver operating characteristic (ROC) curve analysis in the 43 primary ccRCCs (16). To determine the Gpnmb score, the sensitivity and specificity for each outcome in the study were plotted, thus generating various ROC curves. The score was selected as the cutoff value closest to the points of maximum sensitivity and maximum specificity. The tumors designated as having 'low expression' of Gpnmb were those with scores below or equal to the cutoff value, whereas tumors with 'high expression' were those with scores above the cutoff value. To facilitate ROC curve analysis, the cases were dichotomized as follows: death vs. others (censored, alive).

Follow-up. All patients provided follow-up records for more than four years. Following completion of therapy, the patients were observed at 3-month intervals during the first three years and at 6-month intervals thereafter. Overall survival (OS) was defined as the time from diagnosis to the date at which the patient succumbed to the disease or the latest date of census if the patient was still alive.

Statistical analysis. The mean scores and the differences between primary and metastatic tumors were analyzed using paired t-tests. For survival analysis, the optimal cutoff point for Gpnmb expression was obtained by ROC analysis of the primary ccRCC set $(n=43)$. For validation, the correlation between Gpnmb expression, which was classified using a ROC analysis-generated cutoff point, and OS was evaluated in this set. The $\chi^{2}$ test or Fisher's exact test was employed to evaluate 
A
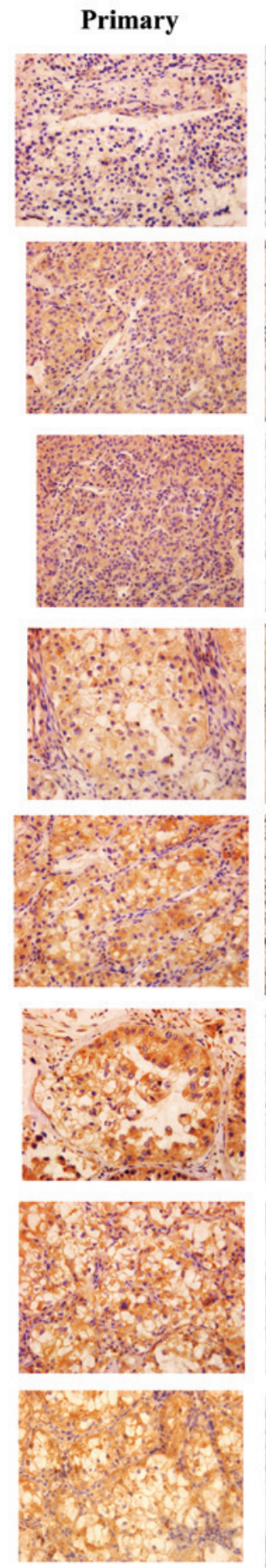

Metastasis
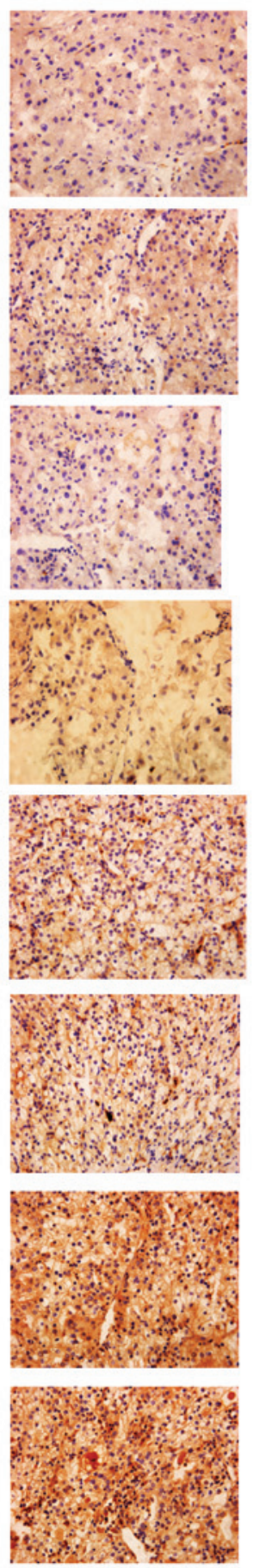

Score

1

2

3

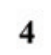

6

8

12

B

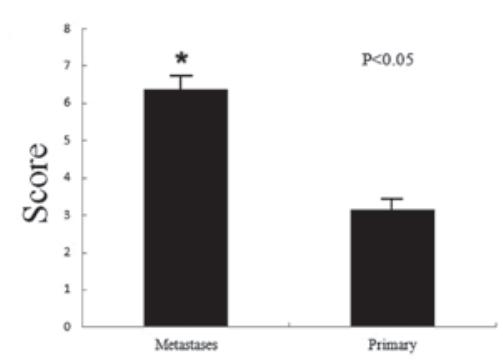

Figure 1. Glycoprotein non-metastatic melanoma protein B (Gpnmb) expression in the 12 paired clear-cell renal cell carcinomas. (A) The upper panel shows examples of a histospot from metastatic specimens with different scores and the lower panel shows the primary tumors (magnification, x200). (B) The difference in mean scores of Gpnmb expression between primary and metastatic tumors was statistically significant $(\mathrm{P}<0.05)$.

the correlation between Gpnmb expression and clinicopathological variables. The multivariate Cox proportional-hazards model was utilized to estimate the hazard ratios for patient outcome. The correlations between Gpnmb expression and OS were determined by Kaplan-Meier analysis. Log-rank tests were performed to assess differences in survival probabilities among patient subsets. All P-values were two-sided, and $\mathrm{P}<0.05$ was considered to indicate a statistically significant difference. Statistical analysis was performed using SPSS 17.0 (SPSS, Inc., Chicago, IL, USA).

\section{Results}

Gpnmb expression in the two ccRCC sets. The clinical features of 12 pairs of primary ccRCCs and matched metastatic tissues from patients, including age, gender, histologic differentiation and Fuhrman grade are summarized in Table I. Gpnmb expression was examined in 12 pairs of primary ccRCCs and matched metastatic tissues by immunohistochemistry and Gpnmb was found to be strongly expressed in the metastatic lesions, with significant upregulation in metastatic RCCs compared with matched primary tissue (Fig. 1, $\mathrm{P}=0.036$ ). To further assess survival, ROC curve analysis was employed to determine the cutoff score for Gpnmb expression. The Gpnmb cutoff score for OS in 43 primary ccRCCs was $5(\mathrm{P}<0.05$, Fig. 2; the cutoff value closest to the points of maximum sensitivity, 0.929 , and maximum specificity, 0.448). Therefore, a Gpnmb expression score of $5(>5 \mathrm{vs} . \leq 5)$ was selected as the uniform cutoff point for survival analysis in the tested set.

Correlation between Gpnmb expression and ccRCC patient clinicopathologic features. In the cohort of 43 primary ccRCCs, high Gpnmb expression was observed in 29/43 (67.44\%) of the ccRCC samples. A correlation analysis revealed that there was no significant correlation identified between Gpnmb expression and clinicopathological features, including patient gender, age, clinical stage and Fuhrman grade $(\mathrm{P}>0.05$, Table II).

Gpnmb expression and survival analysis: Univariate survival analysis. Kaplan-Meier analysis demonstrated that elevated Gpnmb expression predicted inferior OS in the 43 primary $\operatorname{ccRCCs}(\mathrm{P}=0.02$, Fig. 3$)$.

Independent prognostic factors for $c c R C C$. As the variables that were observed to have a prognostic influence on ccRCC patients in the univariate analyses may be correlated, the expression of Gpnmb, clinical stage and Fuhrman grade, which were significant in the univariate analyses in the two cohorts, were further evaluated in a multivariate analysis. Gpnmb expression in ccRCC tissues was found to represent an independent prognostic factor for poor OS in the 43 primary ccRCCs (Table III).

\section{Discussion}

Previous studies have identified gene expression changes resulting in aggressive behavior or metastatic potential in $\operatorname{RCC}(17,18)$. In addition, a bone-seeking clone of metastatic ccRCC was developed (19), which demonstrated that the 
Table II. Association between Gpnmb expression and patient characteristics in renal cell carcinoma.

\begin{tabular}{lccc}
\hline Variable & $\mathrm{n}$ & Positive n (\%) & P-value $^{\mathrm{a}}$ \\
\hline $\begin{array}{l}\text { Gender } \\
\text { Male }\end{array}$ & 25 & $16(64.0)$ & 0.570 \\
$\quad \begin{array}{l}\text { Female } \\
\text { Age (years) }\end{array}$ & 18 & $13(72.0)$ & \\
$\quad \leq 57$ & 23 & $15(65.2)$ & 0.739 \\
$>57$ & 20 & $14(70.0)$ & \\
$\begin{array}{l}\text { Clinical stage } \\
\quad\end{array}$ & 19 & $11(57.9)$ & 0.235 \\
2,3 & 24 & $18(75.0)$ & \\
Fuhrman grade & & $11(55.0)$ & 0.104 \\
$\leq \mathrm{II}$ & 20 & $18(78.3)$ & \\
$>$ II & 23 & & \\
\hline
\end{tabular}

${ }^{a} \chi^{2}$ test. ${ }^{b}$ Classified by median age (57 years old).

Table III. Results of the multivariate Cox proportional-hazards analysis.

\begin{tabular}{lcc}
\hline Variable & Hazard ratio & P-value \\
\hline Age $\leq 57.00$ years (vs. $>$ 57 years) & 1.037 & 0.950 \\
Male gender (vs. female) & 0.654 & 0.446 \\
Clinical stage III (vs. II+I) & 1.315 & 0.663 \\
Fuhrman grade $\leq$ II (vs. >II) & 3.301 & 0.070 \\
Gpnmb-positive (vs. negative) & 7.719 & 0.049
\end{tabular}

Gpnmb, glycoprotein non-metastatic melanoma protein B.

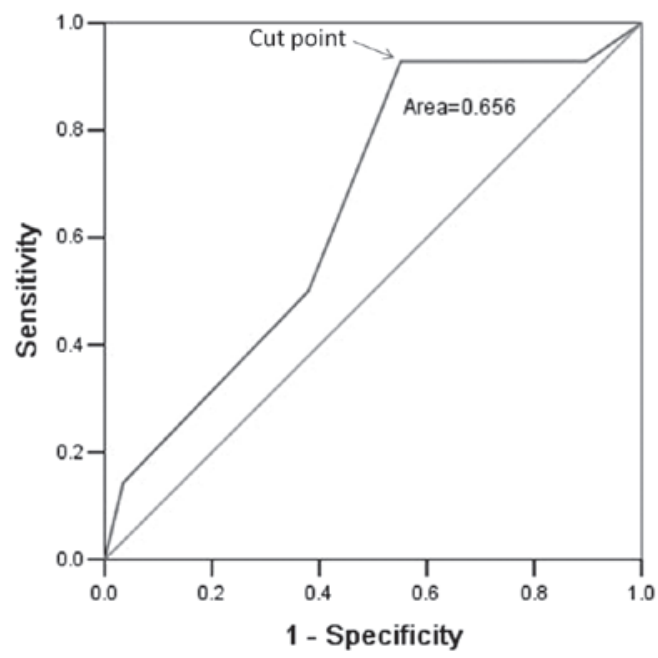

Figure 2. Receiver operating characteristic (ROC) curve analysis of the glycoprotein non-metastatic melanoma protein B (Gpnmb) cutoff score for overall survival in the 34 clear-cell renal cell carcinomas. Each immunohistochemical score, and the sensitivity and specificity for the studied outcome were plotted, thus generating a ROC curve. The Gpnmb cutoff score for overall survival was 5 .

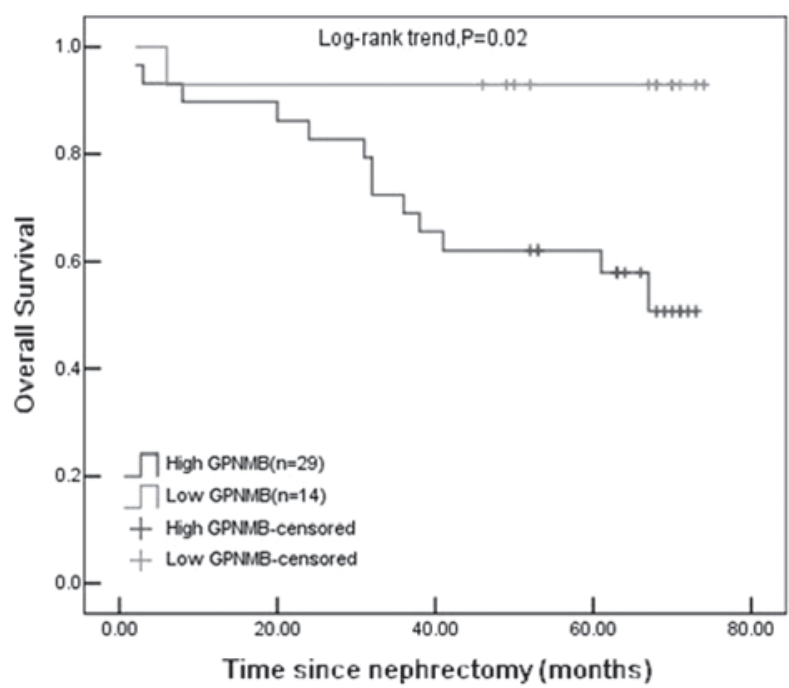

Figure 3. Kaplan-Meier analysis showing that elevated glycoprotein non-metastatic melanoma protein B (Gpnmb) expression strongly predicted inferior overall survival (OS) in the 43 primary clear-cell renal cell carcinomas $(\mathrm{P}=0.02)$. The median duration of OS for patients with low and high expression of Gpnmb was 69.1 and 53.5 months, respectively.

metastasis of ccRCC cells may be the result of a more invasive subclone derived from primary ccRCC cells. A study by Thompson et al (20) showed that high B7-H1 expression is associated with poor prognosis in primary and metastatic $\mathrm{RCC}$; although only one patient was represented in each cohort, metastatic specimens demonstrated higher B7-H1 expression than primary specimens $(54.3 \%$ vs. $44.4 \%$, respectively). Mutant p53 expression was also significantly higher in metastatic tumors compared with primary tumors $(51.8 \%$ vs. $22.8 \%$ ) in a study by Zigeuner et al (21), and p53 overexpression (risk ratio, 2.9; 95\% confidence interval, 1.3-6.7; $\mathrm{P}=0.01$ ) was revealed to be an independent prognostic factor for metastasis-free survival, although the specimens were not matched. In a study of mammalian target of rapamycin- and hypoxia-induced pathway members, which included 135 primary RCCs and 41 unrelated metastases, differential global patterns of expression were measured (22). The levels of p-AKT, p-S6, 4EBP1 and c-myc were higher in metastatic lesions compared with primary tissues.

Differential protein expression between primary ccRCC and subsequent metastases may reveal molecules responsible for metastasis. This may aid in the development of strategies to overcome drug resistance and novel therapies $(1,5,13,14)$. A larger study of 168 metastatic RCC patients who received targeted therapy in situ for their primary tumors reported negligible decreases in the size of the primary tumors (23). Karashima et al (15) reported that the expression levels of a number of angiogenesis-related genes in metastases were relatively higher than in primary tumors. In addition, an in vitro proliferation assay demonstrated the relatively increased resistance of metastatic ccRCC cells to targeted therapy compared with matched primary tumor cells. Clinical observations of discordance in the responses of primary and metastatic tumors also suggest possible differences among stages of tumor biology. One potential weakness of previous studies involves the use of non-matched primary and metastatic tumor tissue. 
For example, metastatic ccRCCs may have different tumor grades and stages in the primary setting when compared with primary ccRCCs, which may have influenced the distribution of the biomarker levels evaluated. In the present study, immunostaining was performed on a tissue set from 12 patients with primary ccRCCs and paired metastases, and the result demonstrated that the expression of Gpnmb was upregulated in metastatic ccRCC compared with primary carcinoma tissue $(\mathrm{P}=0.036)$.

Metastasis is a predominant cause of mortality in clear-cell carcinoma. However, one significant problem in the clinical management of patients presenting with localized ccRCC is the inability to determine tumor aggressiveness and accurately predict which patients are at greater risk of experiencing distant metastases following surgery. A 'metastasis signature' derived from primary RCCs with different prognoses may be used to classify tumors with and without metastases at the time of surgery (1). The purpose of the present study was to identify molecules associated with a 'metastasis signature' indicative of poor prognosis. Gpnmb is a transmembrane glycoprotein that is expressed in various types of cancer and promotes the migration, invasion and metastasis of tumor cells. This protein is also highly expressed in several aggressive types of cancer, including melanoma (a cancer with high propensity for metastasizing to bone), glioma and breast cancer (24-27). Finally, the ectopic expression of Gpnmb is sufficient to enhance invasive phenotypes in vitro and metastatic capabilities in vivo (28). Hong et al (8) previously demonstrated that Gpnmb is important in RCC. The present study assessed differential Gpnmb expression between primary ccRCCs and paired metastases, with the hypothesis that metastasized ccRCC with a high expression of Gpnmb may be the result of a more invasive subclone derived from the primary ccRCC and may be responsible for poor prognosis. Furthermore, the present study assessed the differential prognosis of another 43 primary ccRCCs following radical nephrectomy, which revealed that the high expression of Gpnmb was positively associated with poor survival $(\mathrm{P}=0.02)$. The present study also identified that Gpnmb served as an independent prognostic biomarker for OS in ccRCC $(\mathrm{P}=0.049)$.

In conclusion, these findings provide evidence that the elevated expression of Gpnmb in ccRCC may promote a malignant phenotype with a poor prognosis. To develop an objective Gpnmb cutoff point for the survival analysis, a ROC curve analysis was performed to generate a cutoff score for the 43 ccRCCs. Gpnmb is expressed at the surface of cancer cells (24) but is predominantly expressed intracellularly in normal cells, such as macrophages and melanocytes $(29,30)$. This expression pattern renders Gpnmb particularly attractive for antibody-based therapies since, as a target, the protein is more readily accessible in cancer cells than normal cells, thereby reducing potential complications due to bystander effects. A monoclonal antibody-drug conjugate, CR011-vcMMAE, is currently under development for the treatment of Gpnmb-expressing cancers. The results of the present study identify a molecule that is responsible for metastasis and highlight the requirement for further pathway analysis of metastatic ccRCC to overcome drug resistance and develop novel therapeutic strategies.

\section{References}

1. Tan X, Zhai Y, Chang W, et al: Global analysis of metastasis-associated gene expression in primary cultures from clinical specimens of clear-cell renal-cell carcinoma. Int J Cancer 123: 1080-1088, 2008

2. Fang Y, Wei J, Cao J, et al: Protein expression of ZEB2 in renal cell carcinoma and its prognostic significance in patient survival. PLoS One 8: e62558, 2013.

3. Minn AJ, Kang Y, Serganova I, et al: Distinct organ-specific metastatic potential of individual breast cancer cells and primary tumors. J Clin Invest 115: 44-55, 2005.

4. Nguyen DX and Massagué J: Genetic determinants of cancer metastasis. Nat Rev Genet 8: 341-352, 2007.

5. Laird A, O'Mahony FC, Nanda J, et al: Differential expression of prognostic proteomic markers in primary tumour, venous tumour thrombus and metastatic renal cell cancer tissue and correlation with patient outcome. PLoS One 8: e60483, 2013.

6. Abou Youssiff T, Fahmy MA, Koumakpayi IH, et al: The mammalian target of rapamycin pathway is widely activated without PTEN deletion in renal cell carcinoma metastases. Cancer 117: 290-300, 2011.

7. Reuter VE: The pathology of renal epithelial neoplasms. Semin Oncol 33: 534-543, 2006.

8. Hong SB, Oh H, Valera VA, Baba M, Schmidt LS and Linehan WM: Inactivation of the FLCN tumor suppressor gene induces TFE3 transcriptional activity by increasing its nuclear localization. PLoS One 5: e15793, 2010.

9. Hoashi T, Sato S, Yamaguchi Y, Passeron T, Tamaki K and Hearing VJ: Glycoprotein nonmetastatic melanoma protein b, a melanocytic cell marker, is a melanosome-specific and proteolytically released protein. FASEB J 24: 1616-1629, 2010.

10. Abdelmagid SM, Barbe MF, Hadjiargyrou M, et al: Temporal and spatial expression of osteoactivin during fracture repair. J Cell Biochem 111: 295-309, 2010.

11. Safadi FF, Xu J, Smock SL, Rico MC, Owen TA and Popoff SN: Cloning and characterization of osteoactivin, a novel cDNA expressed in osteoblasts. J Cell Biochem 84: 12-26, 2001.

12. Loftus SK, Antonellis A, Matera I, et al: Gpnmb is a melanoblast-expressed, MITF-dependent gene. Pigment Cell Melanoma Res 22: 99-110, 2009.

13. Tan X, He S, Han Y, et al: Establishment and characterization of clear cell renal cell carcinoma cell lines with different metastatic potential from Chinese patients. Cancer Cell Int 13: 20, 2013.

14. Ohno Y, Izumi M, Tachibana M, et al: Characterization and gene expression analysis of novel matched primary and metastatic renal cell carcinoma cell lines. Oncol Rep 20: 501-509, 2008.

15. Karashima T, Fukuhara H, Tamura K, et al: Expression of angiogenesis-related gene profiles and development of resistance to tyrosine-kinase inhibitor in advanced renal cell carcinoma: characterization of sorafenib-resistant cells derived from a cutaneous metastasis. Int J Urol 20: 923-930, 2013.

16. Saw RP, Morgan M, Koorey D, et al: p53, deleted in colorectal cancer gene, and thymidylate synthase as predictors of histopathologic response and survival in low, locally advanced rectal cancer treated with preoperative adjuvant therapy. Dis Colon Rectum 46: 192-202, 2003.

17. Jones J, Otu H, Spentzos D, et al: Gene signatures of progression and metastasis in renal cell cancer. Clin Cancer Res 11: 5730-5739, 2005.

18. Kosari F, Parker AS, Kube DM, et al: Clear cell renal cell carcinoma: gene expression analyses identify a potential signature for tumor aggressiveness. Clin Cancer Res 11: 5128-5139, 2005.

19. Wang J, Chen A, Yang C, Zeng H, Qi J and Guo FJ: A bone-seeking clone exhibits different biological properties from the ACHN parental human renal cell carcinoma in vivo and in vitro. Oncol Rep 27: 1104-1110, 2012.

20. Thompson RH, Gillett MD, Cheville JC, et al: Costimulatory molecule B7-H1 in primary and metastatic clear cell renal cell carcinoma. Cancer 104: 2084-2091, 2005.

21. Zigeuner R, Ratschek M, Rehak P, Schips L and Langner C: Value of p53 as a prognostic marker in histologic subtypes of renal cell carcinoma: a systematic analysis of primary and metastatic tumor tissue. Urology 63: 651-655, 2004.

22. Schultz L, Chaux A, Albadine R, et al: Immunoexpression status and prognostic value of mTOR and hypoxia-induced pathway members in primary and metastatic clear cell renal cell carcinomas. Am J Surg Pathol 35: 1549-1556, 2011. 
23. Abel EJ, Culp SH, Tannir NM, et al: Primary tumor response to targeted agents in patients with metastatic renal cell carcinoma. Eur Urol 59: 10-15, 2011.

24. Tse KF, Jeffers M, Pollack VA, et al: CR011, a fully human monoclonal antibody-auristatin $\mathrm{E}$ conjugate, for the treatment of melanoma. Clin Cancer Res 12: 1373-1382, 2006.

25. Rich JN, Shi Q, Hjelmeland M, et al: Bone-related genes expressed in advanced malignancies induce invasion and metastasis in a genetically defined human cancer model. J Biol Chem 278: 15951-15957, 2003.

26. Kuan CT, Wakiya K, Dowell JM, et al: Glycoprotein nonmetastatic melanoma protein $\mathrm{B}$, a potential molecular therapeutic target in patients with glioblastoma multiforme. Clin Cancer Res 12: 1970-1982, 2006.

27. Rose AA, Pepin F, Russo C, Abou Khalil JE, Hallett M and Siegel PM: Osteoactivin promotes breast cancer metastasis to bone. Mol Cancer Res 5: 1001-1014, 2007.
28. Onaga M, Ido A, Hasuike S, et al: Osteoactivin expressed during cirrhosis development in rats fed a choline-deficient, L-amino acid-defined diet, accelerates motility of hepatoma cells. J Hepatol 39: 779-785, 2003.

29. Ripoll VM, Irvine KM, Ravasi T, Sweet MJ and Hume DA: Gpnmb is induced in macrophages by IFN- $\gamma$ and lipopolysaccharide and acts as a feedback regulator of proinflammatory responses. J Immunol 178: 6557-6566, 2007.

30. Tomihari M, Hwang SH, Chung JS, Cruz PD Jr and Ariizumi K: Gpnmb is a melanosome-associated glycoprotein that contributes to melanocyte/keratinocyte adhesion in a RGD-dependent fashion. Exp Dermatol 18: 586-595, 2009. 